\title{
E45 USING GENETIC ALGORITHMS TO INVERT NUMERICAL SIMULATIONS
}

\author{
Department of Earth Science and Engineering JONAN CARTER AND CARLOS ROMERO \\ Prince Consort Road, South Kensington, London, SW7 2BP. United Kingdom \\ Email: j.n.carter@ic.ac.uk
}

\begin{abstract}
The use of automated inversion methods to condition numerical reservoir models to both static data (well-logs) and dynamic data (production data) is becoming more important. It is essential that any methodology should be: robust to problems in the numerical simulation; able to handle all of the different classes of variables present; and be efficient both in terms of the number of simulations required and the wall-clock time taken.
\end{abstract}

In this paper we demonstrate that by using a combination of: geostatistical interpolation; a pilotpoint method; and a Genetic Algorithm, one can successfully match a complex Brent sequence reservoir to both production and well-log data. The reservoir is a version of the PUNQ Complex Model, which consists of a realistic Brent sequence, within a complex fault system. There are eleven producers and six injectors, and a total of four years of production was used in the test. The model is characterised by using 513 points for the permeability, the porosity and the net-togross ratio. An additional group of 24 variables are used to control the geostatistical properties, the fault properties, and the well-skin factors, this gives a total of 1563 variables.

The Genetic Algorithm used was the standard generational replacement with elitism method. The structure of the genome and the crossover operators were specially designed for the problem being tackled. Using just 400 numerical simulations it was possible for the methodology to obtain a match similar in quality to that which might be obtained by a good reservoir engineer. Using 20 computers the wall-clock time taken would have been equivalent to the time taken by 20 numerical simulations. The method we have used is robust to loss of simulation results, able to handle many classes of variables simultaneously and is efficient.

\section{Introduction}

The aim of any attempt to invert a numerical model should be that the resulting model will honour all of the available data. Data tend to be available in two forms: measurements that are made on the real world system that we are trying to mimic with our model; and background knowledge that describes the generic nature of the real world system. For a hydrocarbon reservoir the measurements may include: permeability and porosity measurements at particular locations along a well; pressure and flow rate measurements for a well during production; total oil production from the reservoir. The background knowledge may include: statistical relationships between different properties at a single point, spatial-statistical properties for a single property; the structure of geological features. An example of this final type would be that the spatial average of permeability for a continuous sedimentary object does not suddenly change as one crosses the mid-point between two wells.

$8^{\text {th }}$ European Conference on the Mathematics of Oil Recovery - Freiberg, Germany, 3 - 6 September 2002 
When we carry out the inversion of a numerical model, the evaluation of a particular trial model is carried out in three stages. First, we assign values to all of the variables that we are allowed to change during the inversion process, this might be carried out manually or by some optimisation routine. Second, we construct a reservoir model, based on the values of the variables, that is consistent with a selection of the available data, both measurements and background knowledge. Third, having simulated the reservoir's behaviour we compare the model's predictions for all the data not used in the second stage with the actual data. This allows us to assess how well the proposed model honours the actual data. The choice of variables and how knowledge is used in stages two and three are dependent on the reservoir being modelled and the purpose of constructing the model. What is important is that the model building process in stage two, should not introduce unjustified artifacts. In particular one should not have artifacts introduced at stage two by the choice of using a particular optimiser in stage one. The artifacts we have in mind are: those that are caused by using too few variables, and hence the model is not capable of being consistent with the background knowledge; and those that introduce non-geological features.

In the work presented in this paper we started with a given reservoir modelling methodology, and then proceed to construct, and test, an optimisation scheme that does not impose any additional constrains on the model building process. We would not wish to claim that this reservoir modelling methodology used is the best, or most appropriate, that is available. The reservoir modelling methodology is based on the use of pilot-points and geostatistical interpolation. The method is outlined in the next section. The optimisation scheme that we have chosen is a variant of a Genetic Algorithm, and is described in the third section. We did not concur with the view that Genetic Algorithms were not an appropriate optimisation method for reservoir characterisation[7]. The next sections examine the effect of variations in the Genetic Algorithm structure and compares our results with other methods. We finish with some conclusions and observations.

\section{Generating a Reservoir Model}

The reservoir that we have been working with is a variant of the PUNQ Complex Model, whose structure, and well locations are shown in Figure 1. The reservoir is a classic Brent sequence, typical of many found in the North Sea. Details of the sedimentary structure and properties used are given in references [1] and [2]. A complex structure and faulting pattern is then imposed, and the fluid flow properties of the faults are then calculated using the methodology developed by the Fault Analysis Group[3]. In essence the FAG method adjusts the transmissibility of every part of the fault to take account of the total displacement and the amount of clay smearing that has occurred. This results in a much more complex fluid flow behaviour through the fault than is often considered by reservoir engineers. To construct

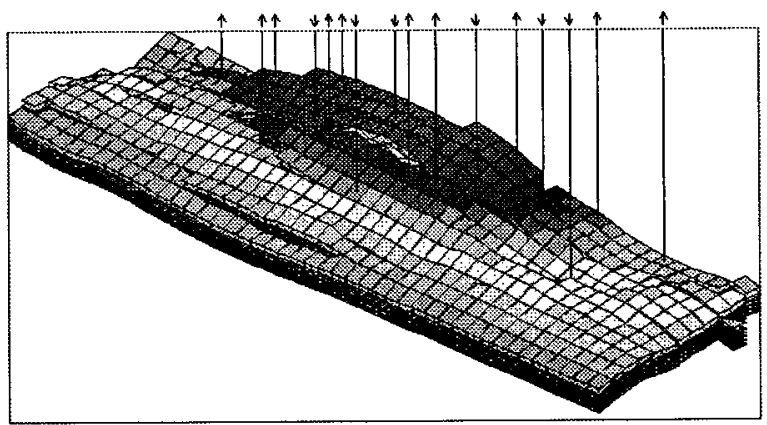

Figure 1 PUNQ Structural Model the reservoir model we need to define in every grid block the permeability, the porosity and the Net-to-Gross ratio. These three property fields are constructed using the pilot-point method and geostatistical interpolation[4][5]. The pilot-points used in this model are shown in Figure 2. On each of the nine active layers of the simulation model, there is a grid of 40 pilot-points, plus pilot-points at each of the wells (generally there are 17 wells in the work presented in this paper). So in total we have 513 pilot-points, this density of points was selected so that most grid blocks 
would be influenced by the values from at least two pilot-points. The complete set of variables also includes: four geostatistical parameters; three fault property parameters; and well-skin factors for each well. In total there are 1563 variables to be defined by the optimisation method for each instance of the numerical model. The building of the reservoir model is summarised in Figure 3.

A consequence of using this methodology for the construction of a numerical reservoir model is that there is not a deterministic link between the values of the variables, and the final reservoir model. Each use of the geostatistical interpolation program will start with a different random number seed, and this will result in a different set of interpolated values, even when the values at the pilot-points are constant. To understand the effect of this one-tomany relationship we carried out the following experiment. First we specified a value for each of the 1563 variables, ensuring that the values chosen are consistent with our geological background knowledge and measurements at the wells. We then construct a numerical simulation model using the process in Figure 3, perform a simulation using ECLIPSE[6] and record monthly values for oil/water flow rates and bottom hole pressures. This model is designated the "truth" model. We then created 20 additional numerical simulation model using the same values for the 1599 variables, but a different random number seed for the gen isticabintempolation: To compare each model with the truth

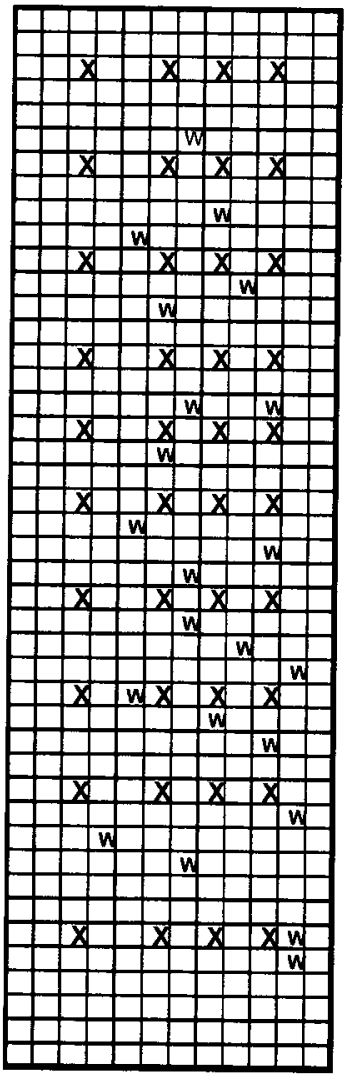

Figure 2 Location of PilotPoints

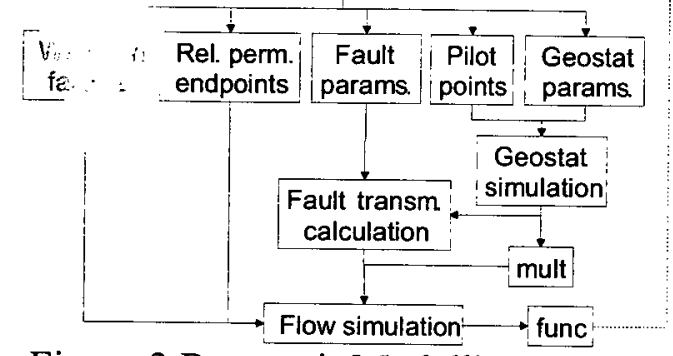

Figure 3 Reservoir Modelling Process model we defined the objective function:

$$
F=\sum_{i}^{n}\left(\frac{o_{i}^{(m)}-o_{i}^{(s)}}{\sigma_{i}}\right)^{2}
$$

where the summation is over all the measurements, the first term is the truth model with added Gaussian noise, the second term is the non-truth case, and this difference is divided by a scaling constant. For the oil/water prodirtion rates the scaling constant was set to $7.5 \%$ of the measurement, which was the sam value as was used when added the noise. For the water injection rate the value was $2 \%$ of measurement. For the bottom hole pressures in both injectors and producers the scaling consiurit had a value of 0.5 Bar. The results are shown in Figure 4, the dotted line is the result of evaluating the objective function for the truth case (ie the effect of the added noise), the other results range from approximately 10 to 720 . These relatively high values give an indication of the difficulty of history matching when using geostatistical interpolation. There can be cases where the optimiser has found approximately the correct values for the variables, but a poor function value is obtained.

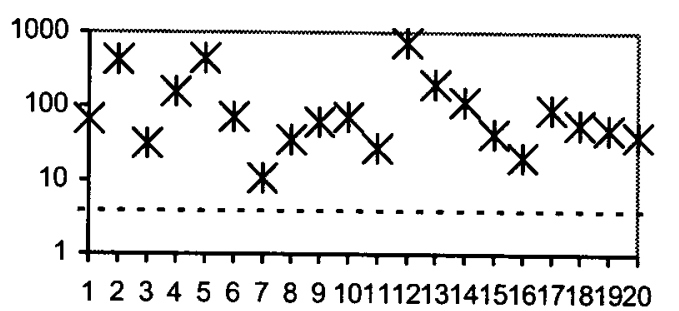

Figure 4 Comparison of Objective Function Values for Similar Parameter Values 


\section{Why Use a Genetic Algorithm?}

There have been many attempts to use optimisation algorithms to automate the selection of values for the variables, so as to match a numerical simulation model to measured data. Each method has advantages and disadvantages, but no single method has met with general acceptance by the reservoir engineering community, a review of these methods can be found elsewhere[2]. It has been suggested[7] that Genetic Algorithms would not be a good choice as an optimiser for this problem. Our reasons for selecting the GA are based on four considerations:

1. The need to be able to handle large numbers of variables, where we do not know which are the most important variables to adjust. Most of the methods we have reviewed would have difficulties in handling $1500+$ variables in a reasonable number of forward simulations (it should be noted that this definition is rather weak and open to a variety of interpretations).

2. The optimisation method must be able to handle the one-to-many mapping that makes any objective surface non-smooth, and which makes it difficult to estimate the gradients of the objective surface.

3. We would like to simultaneously make an assessment of the uncertainty in our results. This requires that we have many relatively independent models close to the global optimum of our objective function, as is possible. It is not essential to exactly identify the global optima during the optimisation process. Many of the methods that have been proposed previously will tend to produce models that are closely related, and which therefore may not be useful for assessing uncertainty. This issue will not be considered further in this paper.

4. The opportunities that arise from the use of coarse-grained parallelism, ie the use of many computers, each of which will run a single forward simulation. Computer clusters of this type are cheap, require only standard software and show a near linear speed-up factor.

We believe that Genetic Algorithms are a good candidate method to address these issues. Other algorithms are also worth considering, we would pick out Evolutionary Algorithms[8] with an MCMC acceptance criteria[9] as an interesting possibility.

In this paper we will only briefly outline the design of our GA, the details of our GA design can be found elsewhere[2][10]. The main structure of the GA is reasonably standard: we use a fixed population size, with generational replacement and single individual elitism; parents are selected by two-person tournaments, with one offspring being produced, parents are then returned to the population for further

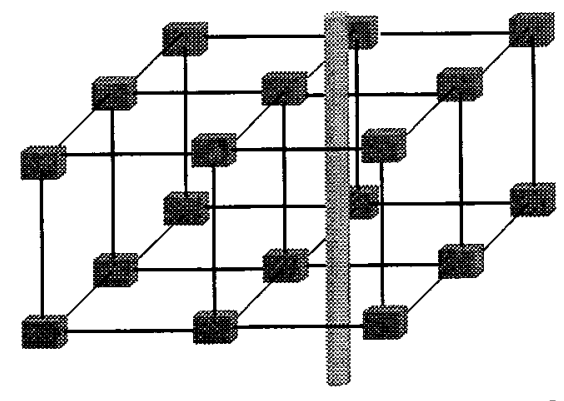
possible tournaments. In a standard GA the variables Figure 5 Three-dimensional Array of Pilot will be stored in a single linear chromosome as either Points.

binary or real numbers. In our GA the variables are stored in several chromosomes. The variables associated with pilot-points are stored as real numbers in three dimensional chromosomes, which include many introns so as to obtain the appropriate spatial relationship (this is illustrated in Figure 5). The other variables are stored as binary numbers in linear chromosomes. The chromosomes are combined and modified using specially designed crossover and mutation operators, which preserve the data at the wells.

In Figure 6 are the results of two optimisation runs. The first uses a different random number seed for each geostatistical interpolation, the second uses a fixed seed in all cases. In both cases the population size is 20 , and we generate 20 generations. The first generation is generated completely randomly, subject to some background knowledge. We can observe that both cases 

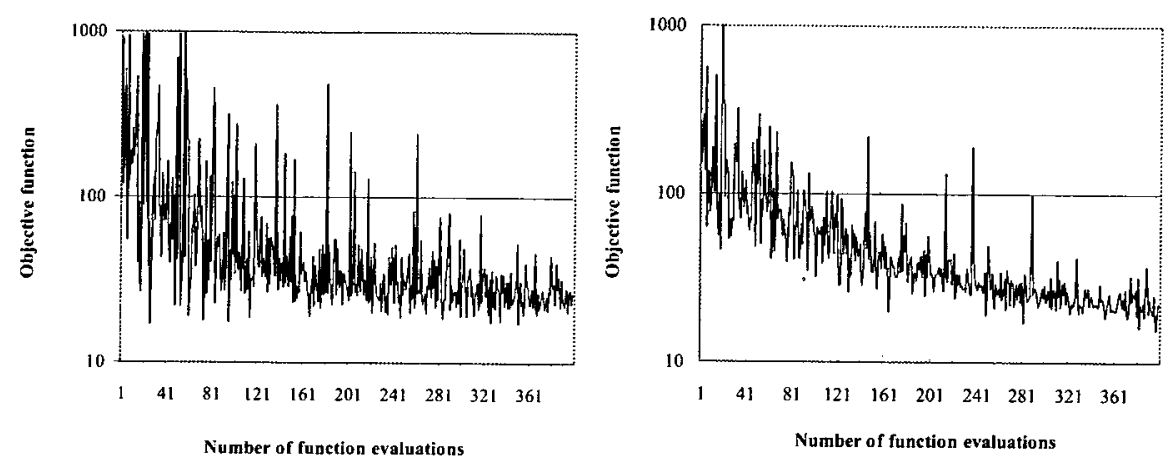

Figure 6 GA Progress Using a Variable Seed, and a Fixed Seed for the Interpolation

are managing to minimise the objective function. The case with the variable random number seed shows much more variability than the fixed seed case. In Figure 7 we can see the pressure matches obtained at two of the production wells. As well as a model from the initial population, and the noisy measurements, models from the $18^{\text {th }}$ and $20^{\text {th }}$ generations are presented. A more general discussion of how well the results match the original production data can be found elsewhere[11].
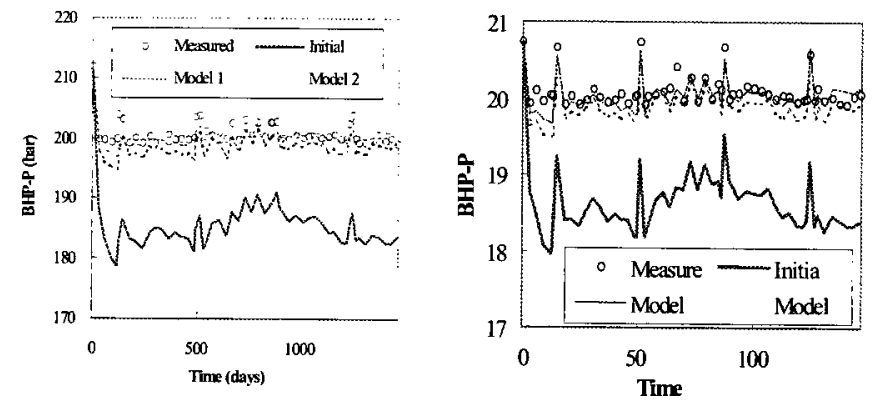

Figure 7 Pressure Matches for Two Producer Wells

\section{What Can We Tune?}

There are many things that can be changed within the overall structure of a Genetic Algorithm. The general structure of the Genetic Algorithm that we have used is given in Figure 8. The things that we might change include: how the variables are encoded, and how they are arranged within chromosomes; how the initial population is generated; how parental selection is carried out; the form of the crossover and mutation operators; the method of culling the population; and the size of the population, the number of offspring created by each pair of parents, the number of offspring per generation. In this paper we will consider two possible changes: the use of real number encoding rather than binary encoding for the non-pilot-point related variables; and the effect of different crossover operators for the pilot-point related variables. For a larger selection of tests, although still far from complete, the reader should consult the original work from which this paper is drawn[2].

\section{Binary vs Real Number Encoding}

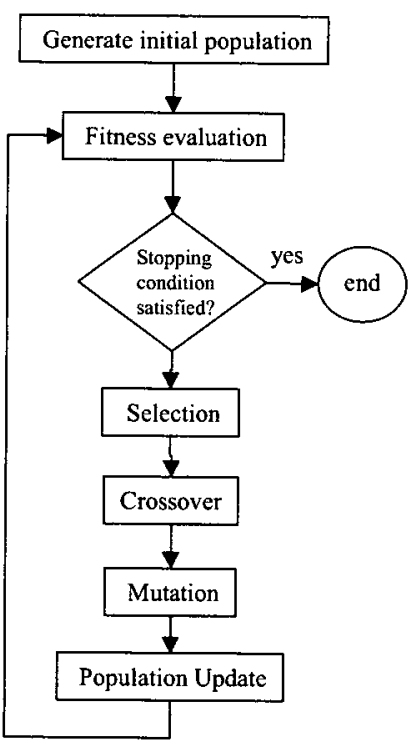

Figure 8 GA Structure

Traditionally within research on the application of Genetic Algorithms, a binary encoding has been used to represent real numbers. In recent years it has been demonstrated that for some problems the direct use of real numbers is beneficial. We have experimented with replacing the binary representation, used for the non-pilot-point parameters, with real numbers. The result of

$8^{\text {th }}$ European Conference on the Mathematics of Oil Recovery - Freiberg, Germany, 3 - 6 September 2002 
this change is shown in Figure 9, where we compare the graphs of the minimum-so-far for the two cases. Based on single optimization runs the introduction of real numbers has resulted in improved performance. If you examine how the variables change during the optimisation process, you find that when a real encoding is used, then some of the variables settle down more quickly. In Figure 10 we compare the well skins for production wells using a two dimensional grey scale plot[12]. Each line represents a different well and we plot the value of the variable for each individual. We can observe that when real number encoding is used many of the

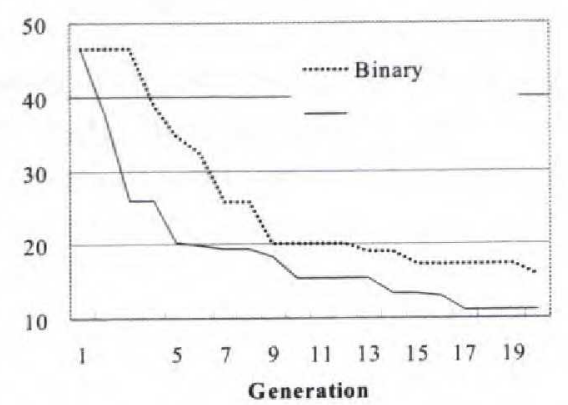

Figure 9 Minimum-so-far variables settle down to fixed values more rapidly. However, in Figure 11 we do not see this effect for the fault parameters (first three lines) and the geostatistical parameters (last four lines). From both figures it can be noted that the optimisation method appears to be identifying different local optima.
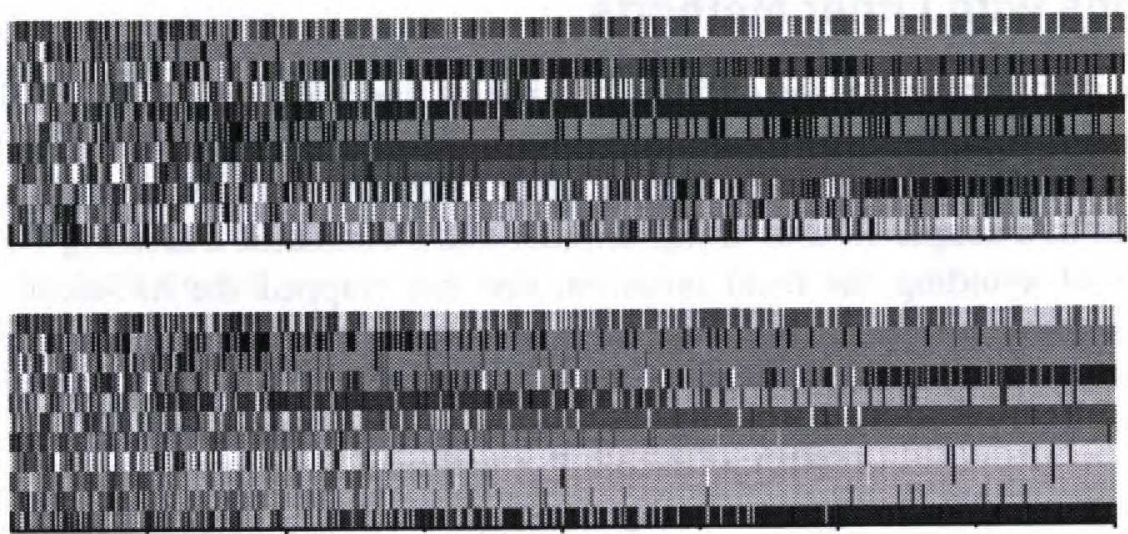

Figure 10 Well-skins for Production Wells (Binary and Real Encodings)

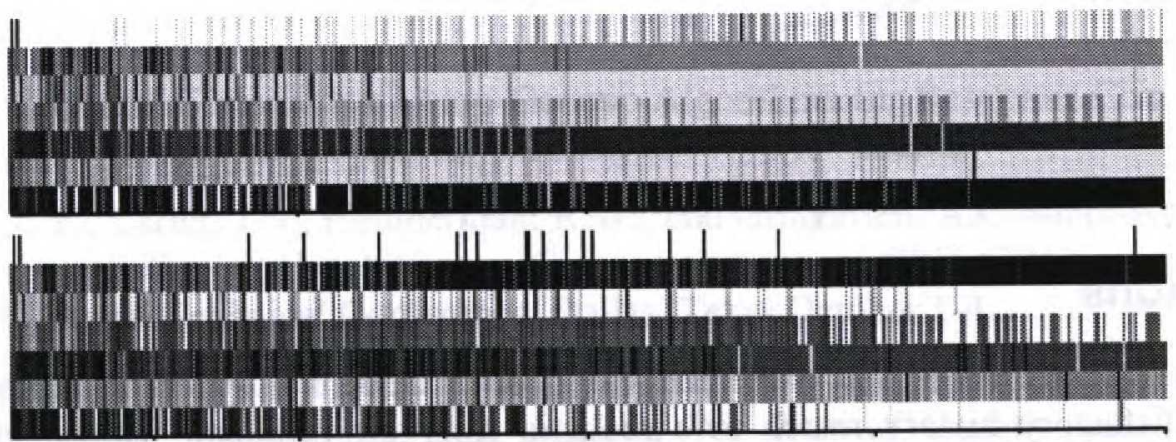

Figure 11 Fault and Geostatistical Parameters (Binary and Real Encodings)

\section{Comparison of Crossover Operators}

We have considered several crossover operators for the pilot-point variables; in this paper we report the results for three of them. The purpose of the crossover operator is to combine the information contained in the genomes of the two parents, to form a new genome which will become the offspring. In the bit-flip operator(C1) we progress in a linear way through the genome, the probability that the next pilot-point variable is copied from the same parent as the last one is $80 \%$, otherwise we copy the variable from the second parent. In the chunks 
operator(C2) all of the pilot-points are copied from the first parent, except for those pilot-points that lie within a randomly chosen box within the reservoir. In the $H$-one-cut operator(C3) the reservoir is randomly divided into upper and lower sections, the upper section is then copied from the first parent and the lower section from the second parent. In Figure 12 we show the generational average and the minimum-so-far for the three cases. There appears to be little advantage to be gained in the choice of the crossover operator.

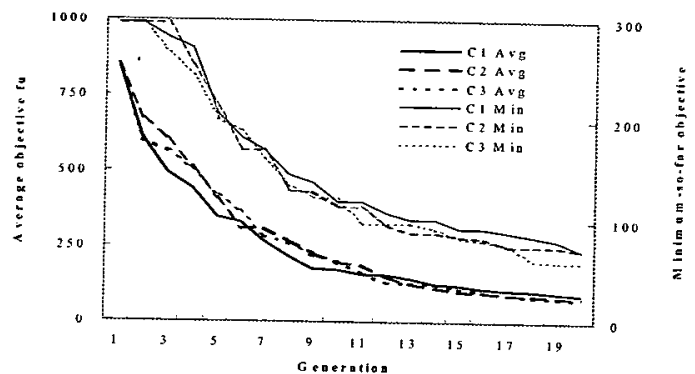

Figure 12 Comparison of Crossover Operators

\section{Comparisons with Other Methods}

Comparisons with other methods is difficult because of the problems caused by the one-to-many mapping. We have made comparisons with three simple algorithms, random search, hill-climber and simulated annealing. The results are shown in Figure 13. The first picture illustrates the relative progress of a simple stochastic hill-climber and a simulated annealing algorithm; we can see the benefit of avoiding the local optimum that has trapped the hill-climber. The second picture plots the minimum-so-far function value against the number of function evaluations. We include a random search, a GA, as well as the hill-climber and simulated annealing optimisers. The result is that the GA appears to out perform the other three optimisers.
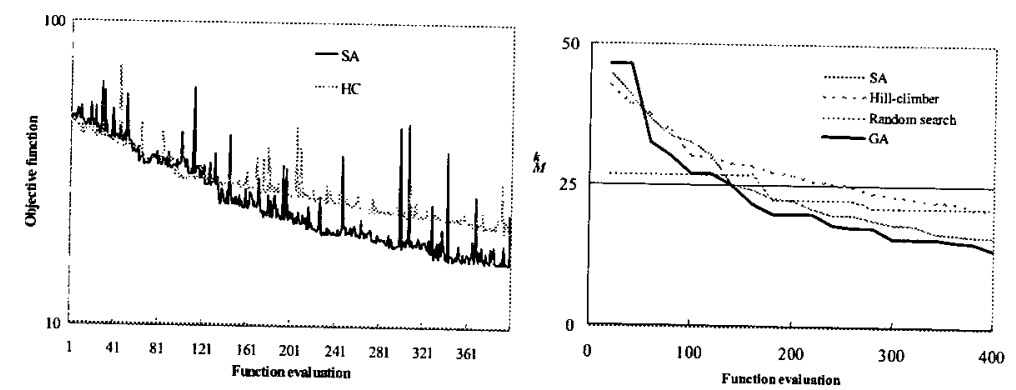

Figure 13 Comparison with Other Optimisers

\section{Conclusions}

The obvious conclusion is that a method based on a Genetic Algorithm seems to be able to obtain a satisfactory history match to a realistic reservoir problem, with a large number of variables, using a reasonable number of forward simulation. The example in this paper could have been obtained in a wall-clock time equivalent to 20 forward simulations, assuming that we had used a cluster of 20 computers to perform the simulations. It would appear that using real number encoding gives a beneficial effect, but that the choice of crossover operator, among those tested, is not important. Future work needs to address the other issues that we used to justify the use of a Genetic Algorithm, and to test in a more systematic way how the Genetic Algorithm should be constructed so as to maximise efficiency. 


\section{References}

[1] Bos, C. (editor), PUNQ project team: Bos, C., Floris, F., Nepveu, M., Roggero, F., Omre, H., Holden, L., Syversveen, A-R., Zimmerman, R., Carter, J., Frandsen, P.E., Bech, N., Geel, C., Walsh, J., Manzocchi, Barker, J., Cuypers, M., Bush, M.: Production Forecasting with Uncertainty Quantification, PUNQ-2, Final Report, report, Netherlands Institute of Applied Geoscience (TNO), NITG-99-255-A, Delft, The Netherlands (December 1999).

[2] Romero,C., A Genetic Algorithm for Reservoir Characterisation using Production Data, $\mathrm{Ph} . \mathrm{D}$. Thesis, University of London, November 2000.

http://www.geocities.com/athens/olympus/8210/thesis.html

[3] Manzocchi, T., Walsh, J.J., Nell, P., and Yielding, G.: Fault Transmissibility Multipliers for Flow Simulation Models, Petroleum Geoscience (1999) 5, 53.

[4] de Marsily, G., Lavedan, G., Boucher, M., and Fasanino, G.: Interpretation of Interference Tests in a Well Field Using Geostatistical Techniques to Fit the Permeability Distribution in a Reservoir Model, Geostatistics for Natural Resources Characterization, Verly, G. et al. (Eds.), D. Reidel Publishing Company (1984) 831.

[5] Bissell, R.C., Dubrule, O., Lamy, P., Swaby, P. and Lepine, O.: Combining Geostatistical Modelling With Gradient Information for History Matching: The Pilot Point Method, paper SPE 38730 presented at the 1997 SPE Annual Technical Conference and Exhibition, San Antonio, Texas, Oct. 5-8.

[6] GeoQuest Reservoir Technologies: ECLIPSE 10098 A (1998).

[7] Oliver, D.S., Reynolds, A.C., Bi, Z, and Abacioglu, Y, Integration of production data into reservoir models, Petroleum Geoscience, 7, S65-S73, (2001).

[8] Yao, X., From Evolutionary Computation to Natural Computation, in Proc $5^{\text {th }}$ Evolutionary/Adaptive Computing in Design and Manufacture, Ed Parmee, I.C., April 2000.

[9] Omre, H., Tjelmeland, H., and Wist, H.T., Uncertainty in history matching, model specification and sampling algorithms. NTNU-Trondheim Internal Report, Statistics Number 6, (1999).

[10] Romero, C.E. and Carter, J.N.: Using Genetic Algorithms for Reservoir Characterisation, Journal of Petroleum Science and Engineering (2001) 31, 113.

[11] Romero, C.E., Carter, J.N., Zimmerman, R.W., and Gringarten, A.C.: Improved Reservoir Characterisation through Evolutionary Computation, paper SPE 62942 presented at the 2000 SPE Annual Technical Conference, Dallas, Texas October 1-4.

[12] Pohlheim, H., Visualization of Evolutionary Algorithms - Set of Standard Techniques and Multidimensional Visualization, Eds Banzhat, W., Proc of Genetic and Evolutionary Computational Conference 1999, Pub Morgan Kaufmann, 1999. 